\title{
Errors in neuroradiology
}

\author{
Ferdinando Caranci $^{1} \cdot$ Enrico Tedeschi $^{1} \cdot$ Giuseppe Leone $^{1} \cdot$ Alfonso Reginelli $^{2}$. \\ Gianluca Gatta $^{2} \cdot$ Antonio Pinto $^{3} \cdot$ Ettore Squillaci $^{4} \cdot$ Francesco Briganti $^{1}$. \\ Luca Brunese ${ }^{5}$
}

Received: 14 April 2015 / Accepted: 25 June 2015 / Published online: 17 July 2015

(C) Italian Society of Medical Radiology 2015

\begin{abstract}
Approximately $4 \%$ of radiologic interpretation in daily practice contains errors and discrepancies that should occur in $2-20 \%$ of reports. Fortunately, most of them are minor degree errors, or if serious, are found and corrected with sufficient promptness; obviously, diagnostic errors become critical when misinterpretation or misidentification should significantly delay medical or surgical treatments. Errors can be summarized into four main categories: observer errors, errors in interpretation, failure to suggest the next appropriate procedure, failure to communicate in a timely and a clinically appropriate manner. Misdiagnosis/ misinterpretation percentage should rise up in emergency setting and in the first moments of the learning curve, as in residency. Para-physiological and pathological pitfalls in neuroradiology include calcification and brain stones, pseudofractures, and enlargement of subarachnoid or epidural spaces, ventricular system abnormalities, vascular system abnormalities, intracranial lesions or pseudolesions, and finally neuroradiological emergencies. In order to minimize
\end{abstract}

Ferdinando Caranci

ferdinandocaranci@libero.it

1 Unit of Neuroradiology, Department of Advanced Biomedical Sciences, Federico II University of Naples, Naples, Italy

2 Department of Internal and Experimental Medicine, Magrassi-Lanzara, Institute of Radiology, Second University of Naples, Caserta, Italy

3 Department of Radiology, Cardarelli Hospital, Naples, Italy

4 Department of Diagnostic Imaging, Molecular Imaging, Interventional Radiology and Radiotherapy, University of Rome Tor Vergata, Rome, Italy

5 Department of Health Science, University of Molise, Campobasso, Italy the possibility of error, it is important to be aware of various presentations of pathology, obtain clinical information, know current practice guidelines, review after interpreting a diagnostic study, suggest follow-up studies when appropriate, communicate significant abnormal findings appropriately and in a timely fashion directly with the treatment team.

Keywords Neuroradiology $\cdot$ Errors $\cdot \mathrm{CT} \cdot \mathrm{MRI}$

\section{Introduction}

Diagnostic errors are important in all branches of medicine but they are critical in diagnostic radiology and neuroradiology, where misinterpretation or misidentification may significantly delay medical or surgical treatments.

As defined by Borgstede et al. [1] approximately $4 \%$ of radiologic interpretations in daily practice contain errors, and discrepancies occur in 2-20\% of reports [2]. Fortunately, most of them are minor degree errors, or, if serious, are found and corrected with sufficient promptness.

Errors should be distinguished into two categories, perceptual and cognitive: in perceptual errors abnormality is not perceived, and in cognitive errors the abnormality is perceived but misinterpreted [3, 4].

Moreover, error does not equal negligence; in fact negligence occurs when the degree of error exceeds an accepted standard. Although human error is inevitable in medicine, including neuroradiology, it is important to distinguish medical errors from medical malpractice; medical error is the failure of a planned action to be completed as intended; medical malpractice is defined as a failure of the physician to exercise that degree of skill and knowledge commonly 
applied under all the circumstances in the community, resulting in injury to the patient [3].

In order to minimize the possibility of error, it is important to be aware of the various possible presentations of pathology, obtain clinical information, know current practice guidelines, review after interpreting a diagnostic study, suggest follow-up studies when appropriate, communicate significant abnormal findings appropriately and in a timely fashion directly with the treatment team.

\section{Sources of errors in neuroradiology}

Medical-legal literature divides missed radiologic findings into errors due to negligence and errors not due to negligence. The legal basis for negligence involves a breach of the standard of care, which is usually defined as being the use of the same degree of knowledge, skill, and ability as an ordinary careful physician would exercise under similar circumstances [3-5].

Errors could be summarized in four main categories: observer errors, errors in interpretation, failure to suggest the next appropriate procedure, failure to communicate in a timely and a clinically appropriate manner $[6,7]$.

Kundel et al. [8] described three types of observer error: scanning, recognition and decision-making error. Scanning error is the result of failure of the radiologist/neuroradiologist to fixate his/her attention in the area of the lesion. Recognition error involves fixating in the territory of the lesion yet failing to detect the lesion. The most common error is decision-making error, which accounted for approximately $45 \%$ of observer error in the study by Kundel et al. [8]. This error is due to incorrect interpretation of a malignant lesion as a normal structure after detection.

There are many reasons why radiologists make errors in identifying and interpreting abnormalities. Factors such as clinical history, presence or absence of previous studies, index of suspicion, the reading room environment, and the level of vigilance of the interpreter are various sources of error [6].

Failure to suggest the next appropriate procedure is an extremely important error. In fact, follow-up or additional diagnostic studies to clarify or confirm the impression should be suggested when appropriate to the neuroradiologist's own judgment [7].

Errors in communication are the fourth most frequent allegation against radiologists in medical malpractice claims [9]. In fact, in the official interpretation (a final written report), the radiologist is responsible for communicating all the findings directly to the referring physician.

Reenfrew et al. [10] have classified the principal cause of radiological errors. It includes complacency, i.e., when the finding is appreciated but attributed to the wrong

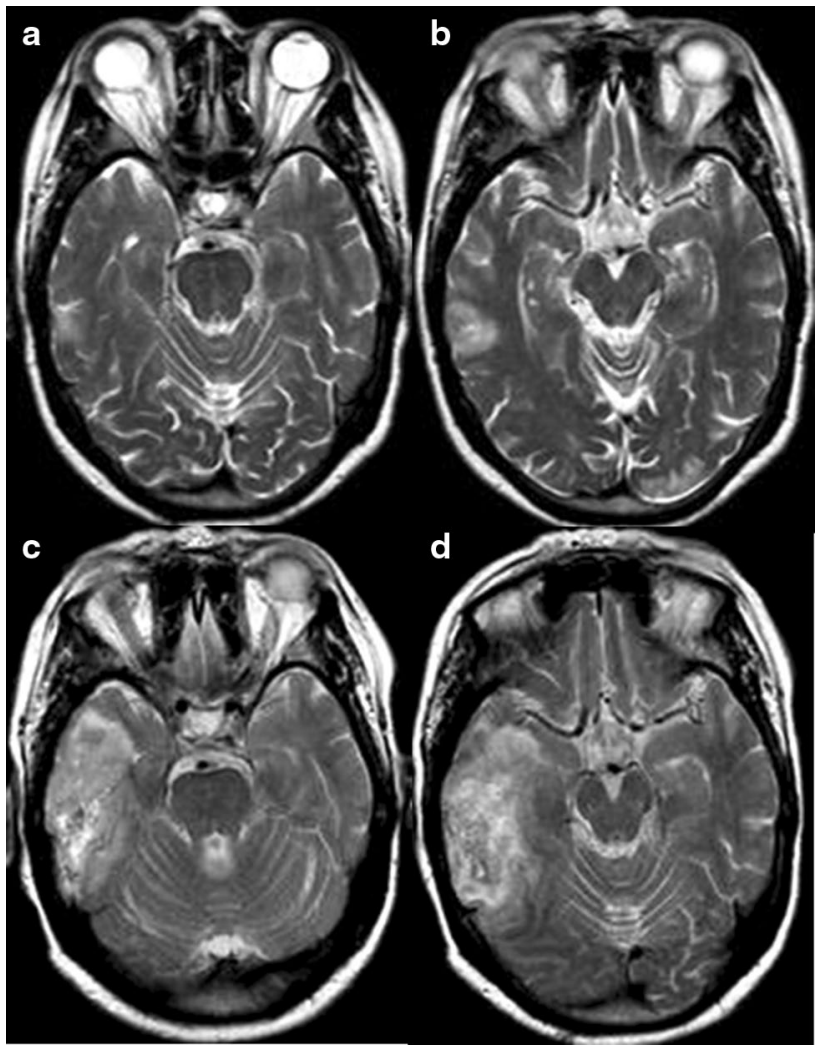

Fig. 1 Faulty reasoning. a, b Axial T2-weighted images: presence of a hyperintense cortico-subortical lesion in the right temporal lobe, interpreted as a subacute ischemia. c, d Axial T2-weighted images: 4 months later, marked enlargement of the lesion, with inhomogeneous structure (high-grade glioma)

cause; faulty reasoning, when the finding is appreciated and interpreted as abnormal, but attributed to the wrong cause (Fig. 1); lack of knowledge on the part of the viewer; under-reading; and poor communication, i.e., when the lesion is identified and interpreted correctly, but the message failed to reach the relevant clinician [10]. Moreover, important mistakes are the missed communication of findings that required urgent contact with the patient's primary physician for possible change in treatment.

Workload can be a factor in increasing the likelihood of errors in radiology reporting [4], as well as short experience of staff, inadequate equipment, inadequacy of clinical information, inappropriate expectations of the capability of a particular radiologic technique, unavailability of previous studies or reports for comparison. Adoption of standard imaging protocols may reduce the likelihood of error or discrepancy in some areas of neuroradiology practice, especially in MRI $[6,7,11]$.

Moreover, diagnostic accuracy in neurology frequently depends on clinical assessment and neuroimaging interpretation. McCarron et al. have analyzed neuroimaging discrepancy rates in reported findings between general 
radiologists and neuroradiologists relative to patients from a district general hospital [12]. Discrepancies in the primary diagnosis/abnormality were identified in $14.2 \%$ of the patients. Incidental findings such as brain atrophy, pineal or arachnoid cysts, and differences in differential diagnoses were all categorized as secondary findings and differed in $21.5 \%$ [12].

In the literature many reports show low discrepancy rates between general or trainee radiologists and neuroradiologists [13]. In a large study of over 2000 scans, clinically relevant discrepancies were reported in just $1.8 \%$ among faculty neuroradiologists. Clinically relevant discrepancies have a similarly low frequency rate of less than $2 \%$ in many radiology subspecialties [14].

Babiarz et al. [15] performed a quality control study of image interpretation discrepancies in academic neuroradiology and found $87.6 \%$ agreements with the original report in 1000 randomly analyzed CT and MR studies. Discrepancies were observed for neoplastic and vascular pathology.

\section{Resident's errors}

In emergency setting and in the first periods of the learning curve, as in residency, misdiagnosis/misinterpretation percentage should rise up. A recent study has evaluated common misinterpretations of head CT scans by radiology residents in a level I trauma center [16]: the overall misinterpretation rate was $41 \%$. The most commonly missed pathologies were chronic infarcts, hypodense lesions, and mucosal thickening in the paranasal sinuses. Anatomic misinterpretations were defined as errors in identifying the correct anatomic location of the pathology. For example, a brain contusion could be identified on the wrong brain lobe, or a subdural location of a hematoma could be confused with an epidural location. The most common sites for misdiagnosis were brain lobes, sinuses, and deep brain structures. The highest percentage of misinterpretation occurred between 2.30 p.m. and 8 p.m. and the lowest between midnight and 8 a.m. Considering only the important diagnosis requiring urgent treatment, the overall percentage of errors was $4.7 \%$ [16]. It is interesting to note that a recent study by Walls et al. [17] showed that the period with the highest percentage of mistakes was between 0400 and 0800 , the last hours of the shift, which the authors attributed to fatigue. Multiple previous studies found an association between rate of discrepancies and resident training level, with preliminary reports issued by first-year residents being more often discrepant $[18,19]$. This finding is not surprising, as first-year residents are by definition less experienced.

CT exams represent the modality of choice in the emergency room setting for on-call neuroradiology studies, and studies have shown low miss rates of significant lesions [18]. MR imaging is going to be an expected level of radiology care for the diagnosis and treatment of emergency room patients; therefore, it becomes imperative that on-call radiology residents in academic centers be trained to meet this emerging standard.

For MR exams, Filippi et al. [20] have analyzed 361 spine MR imaging and MR angiographic examinations that were ordered emergently after hours and given preliminary interpretation by radiology residents. Discrepancies between the interpretations of radiology residents and the final reports of attending neuroradiologists were classified as either false-negative (FN, failure to recognize abnormalities) or false-positive (FP, misinterpreting normal images as abnormal). Overall, the agreement rate was $92.8 \%$, the overall discrepancy rate was $7.2 \%$, the major disagreement rate was $4.2 \%$, and the minor disagreement rate was $2.2 \%$. Misinterpretations among first-year residents on call were significant when compared with more seniorlevel residents. There were $23 \mathrm{FN}$ interpretations. The most common misses were acute stroke, aneurysm, vascular occlusion, and disk herniation. There were only three FP interpretations (misdiagnoses of syrinx, arachnoiditis, and acute infarct) [20]. Analyzing this series, discrepancy rates between radiology residents and attending neuroradiologists on emergent MR imaging studies, including brain and spine MR examinations and neck and circle of Willis MR angiograms, were low, and there was no adverse clinical outcome as a result of discrepant interpretations.

\section{Para-physiological and pathological pitfalls}

\section{Calcification and brain stones}

Vascular or choroid plexus calcifications are frequently seen in the brain structures, commonly in the lateral ventricles but also in the fourth ventricle, and should not be confused with blood or even tumor. Temporal horns calcifications are sometimes indistinguishable from intraparenchymal ones; calcifications of the choroid plexus in the roof of the third ventricle or in the region of the foramen of Monro may resemble colloid cysts or calcified neurocysticercosis [21].

Large intracranial calcifications are occasionally encountered in routine CT scans of the brain. Based on location, brain stones can be classified as extra- or intraaxial. Extra-axial brain stones comprise tumors and exaggerated physiological calcifications. Intra-axial brain stones can further be classified according to etiology, namely neoplastic, vascular, infectious, congenital, and endocrine/ metabolic. Imaging findings combined with essential clinical information can help in narrowing the differential 
diagnosis, determining disease state, and evaluating effect of therapy. CT scan is the mainstay in identifying and characterising brain stones. Certain MRI sequences (gradient echo T2* and susceptibility-weighted imaging) are considered adjunctive [22].

\section{Pseudofractures and enlargement of subarachnoid or epidural spaces}

Sutures, nerve canals can be interpreted as false images of pseudofracture; the asymmetry of the jugular fossa or the internal auditory canal may suggest possible tumors [23]. Subarachnoid and epidural space may be enlarged without clinical consequences. Mega cisterna magna, arachnoid cysts, and Virchow-Robin perivascular spaces can occur frequently and they should not be confused with pathological images (Fig. 2) [24].

\section{Ventricular system abnormalities}

Ventricular abnormalities comprise lateral ventricle asymmetry (left ventricle is usually larger than right) with significant bulging of the septum pellucidum, ventricular coarctation (which usually occurs in the frontal or occipital horns due to fusion of the ependymal walls), cavum septum pellucidum/cavum vergae/cavum velum interpositum, cerebrospinal fluid collections that simulate the appearance of cysts, and finally ependymitis granularis or chronic interstitial edema around the ventricles, asymmetric anterior horns mimicking demyelinating lesions [21,25].

\section{Vascular system abnormalities}

Multidetector CT angiography is used in many institutions for the initial evaluation of the cerebral circulation in acute stroke and subarachnoid hemorrhage.

Knowledge of the main vascular anatomic variants (fenestrations, duplications, and persistent fetal arteries) may aid in surgical planning: for example, the preoperative detection of a medial or intrasellar persistent trigeminal artery may help surgeons to avoid a potentially life-threatening hemorrhage in a patient undergoing trans-sphenoidal surgery for pituitary adenoma [26].

\section{Intracranial lesion or pseudolesion}

The differentiation between intra-axial versus extra-axial lesions is usually routinary, but sometimes it can be very difficult [27]. Extra-axial lesions, particularly meningiomas, commonly show a broad dural base or a dural tail of enhancement [21]; this may also occur in other extra-axial
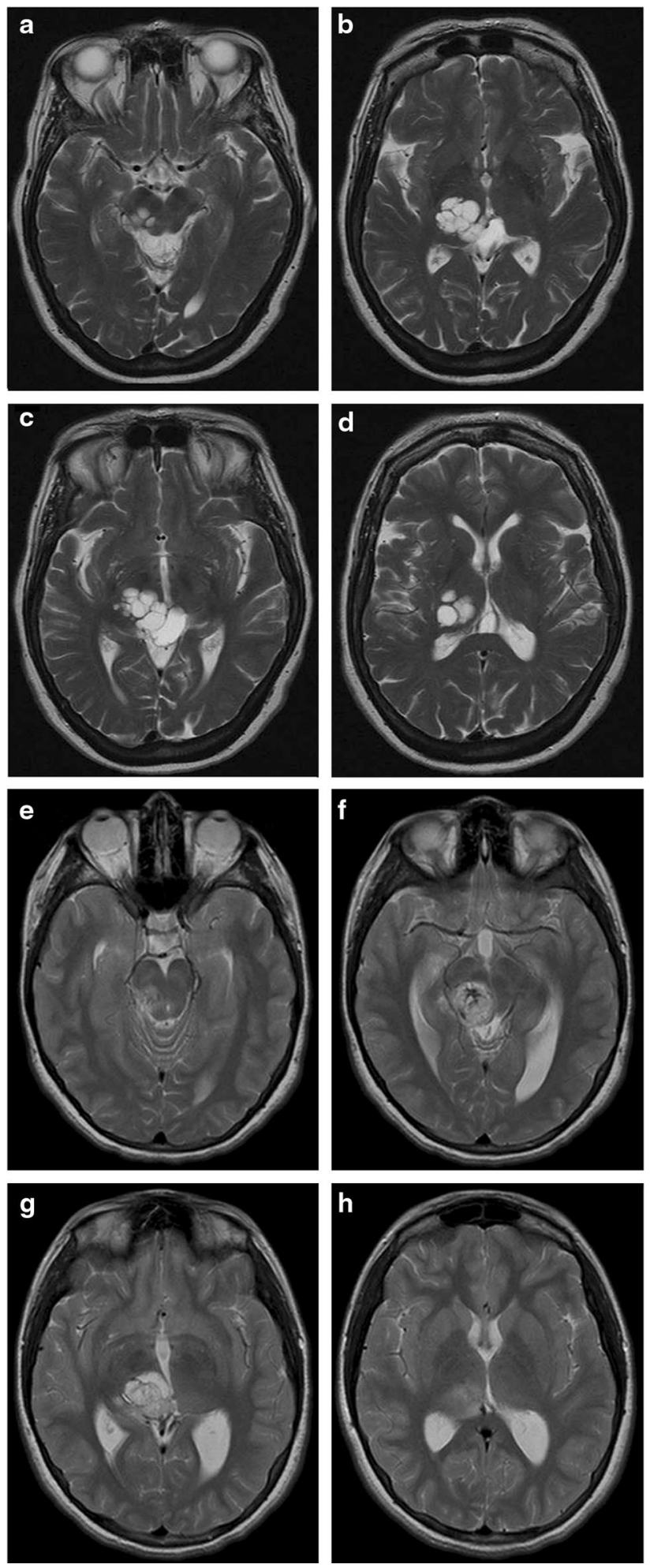

Fig. 2 Virchow-Robin perivascular spaces and differential diagnosis. a-d Axial T2-weighted images: giant perivascular spaces in thalamomesencefalic location, characterized by liquoral signal. e-h Axial T2-weighted images: presence of a glial tumor in the same location, characterized by a non-homogeneous signal 

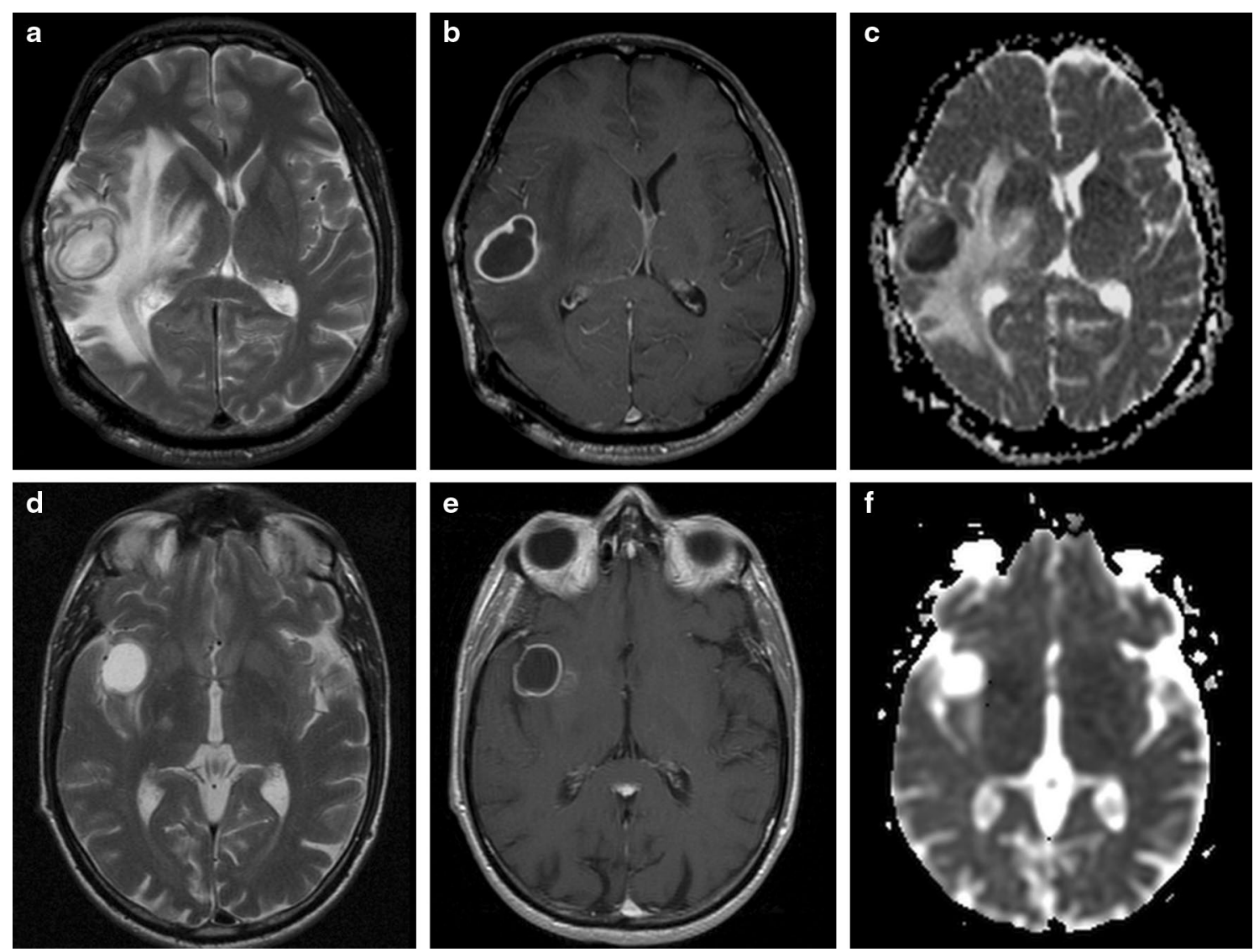

Fig. 3 Brain abscess and glial neoplasm (differential diagnosis). a-c Axial T2 weighted, contrast-enhanced T1 weighted, diffusionweighted images: ring-enhancing, liquified lesion, characterized by restricted diffusion, associated with extensive peripheral vasogenic

tumors, although less commonly. Bony change is another sign of an extra-axial origin, usually confirmed by CT.

Another important consideration is the effect on the surrounding structures: primary brain tumors often have less mass effect for their size than you would expect, due to their infiltrative growth; this is not the case with metastases and extra-axial tumors, which have more mass effect due to their expansive growth.

Many non-tumoral lesions can mimic a brain tumor (Fig. 3): abscesses can mimic metastases; multiple sclerosis can present as a mass-like lesion with enhancement, also known as tumefactive multiple sclerosis; in the parasellar region one should always consider the possibility of aneurysm; infections and vascular lesions can also mimic a CNS tumor [21, 28-30].

Knowledge of the patterns and mechanisms of contrast medium enhancement facilitate the radiologic differential diagnosis. Extra-axial enhancing lesions include primary neoplasms (meningioma), granulomatous disease (sarcoid), and metastases. Linear pachymeningeal (dura-arachnoid) enhancement occurs after surgery and with spontaneous edema. d-f Axial T2 weighted, contrast-enhanced T1 weighted, diffusion weighted images: ring-enhancing, colliquative lesion, characterized by elevated diffusion

intracranial hypotension; leptomeningeal (pia-arachnoid) enhancement is present in meningitis and meningoencephalitis; superficial gyral enhancement is seen after reperfusion in cerebral ischemia, during the healing phase of cerebral infarction, and with encephalitis; nodular subcortical enhancement is typical for hematogenous dissemination and may be neoplastic (metastases) or infectious (septic emboli). Deeper lesions may form rings or affect the ventricular margins; smooth and thin ring enhancement is typical of an organizing abscess or necrotic neoplasm [31].

Finally, technical deficiencies such as patient motion artifacts, beam hardening artifacts, position asymmetries or partial volume averaging effects may mislead the radiologist and create false images of pathology. Pseudolesions in the brain parenchyma as the temporal lobe hypodensity or some posterior fossa abnormalities in the cerebellum may be related to beam hardening artifacts; differential diagnosis should include cerebral contusions and white matter injuries. The vermian pseudotumor is the visual effect of an hyperdense structure surrounded by the fourth ventricle and the paravermian cistern, which are hypodense structures [32]. 


\section{Trauma and neuroradiological emergencies}

Error can increase the rate of mortality and morbidity in the managing of traumatized patients and other neuroradiological emergencies [33]. In the cranial-cervical region, two frequent errors in the radiological procedure may result in a serious increase in morbidity and mortality: understaging of the supra- aortic trunks lesions and overlooking the cause of the intraparenchymal hematomas of the brain. The first type can be avoided by extending the study of the arterial chest- abdominal phase to the neck, as the frequency of traumatic injuries in this vascular district is high enough (1-5\%), and with obviously serious consequences in terms of morbidity and mortality, to warrant assessment [34]. In the second, an intraparenchymal hematoma in a polytraumatized patient is often indicative of either an arteriovenous malformation or the spontaneous bleeding of an intracranial aneurysm. Such cases are best evaluated by integrating the pre-contrast study of the skull with a cerebral CT- or MR-angiography [35].

The management of cervical spine injuries remains complex and controversial. Clinical status, the type of trauma, and the various trauma risk factors are important elements that must be considered in the choice of an imaging modality. Injuries of the vertebral column are involved in $10 \%$ of all misdiagnoses occurring in the initial phase. Such injuries are much more common at the cranio-cervical junction (40-50\%) and at the cervicaldorsal transition [36].

In 2009, the American College of Radiologists (ACR) recommended multidetector computed tomography (MDCT) with multiplanar reconstruction (MPR) as the method of choice in suspected spinal trauma, replacing traditional imaging studies for patients who show a suspicious clinical framework according to the criteria of NEXUS or the Canadian cervical spine rules [2-37].

\section{How to reduce errors in Neuroradiology}

Diagnostic errors can be reduced by improvements both in individual knowledge and in organization. Better system organization consists in improvements of working conditions and in the time available for reporting, of equipment modifications to prevent accidental error, in double reporting, and in regular dialogue between clinicians and radiologists [38]. Diffusion of a peer review process in diagnostic neuroradiology is one approach to respond to this need. Double-reading has been proposed for interpretation of CT angiography of the head and the neck [39]. In this study, discrepancies were discovered in 20 studies, corresponding to $4.0 \%$. This idea is worth mentioning, but in reality the workload issues faced by most radiology departments are such that double reporting is beyond their resources. Some departments have integrated peer review into their daily clinical workflow by providing previous interpretations with every new study and including a checkbox for the interpreting radiologists to indicate whether they agree with the previous interpretations and, if not, a text box to indicate why they disagree. Discrepancies are reported to the original radiologists by e-mail. This system minimizes the time required of the reviewing radiologists [40].

Although a missed diagnosis or other forms of error may be the most feared event for a radiologist, it can also be one of the best opportunities for learning. Learning from errors requires a critical appraisal of our own practice and the implementation of change to enhance performance levels.

Educational programs, morbidity meetings, and a comprehensive and respected root cause analysis process are important for decreasing the likelihood of future diagnostic errors.

\section{Conclusion}

The main reason for studying medical errors is to try to prevent them. Retrospective analysis of cases in which an error is felt to have arisen has educational benefit, and an appreciation of the error along with the identification of its possible causal factors enables the appropriate modification such that in the future similar errors might be avoided. Although a missed diagnosis or other form of error may be the most feared event for a neuroradiologist, it can also be one of the best opportunities for learning.

Diagnostic errors fall into recurrent patterns. Error traps need to be uncovered and highlighted to prevent repetition of the same mistakes. Learning from errors requires a critical appraisal of our own practice and the implementation of change to enhance performance levels. Wherever errors are made, they should be seen as learning opportunities, not just individually, but corporately too: for neuroradiology and medical colleagues, radiology trainees, and students.

Identification and reduction of diagnostic error provides a measure of the efficacy of the healthcare system, as it reduces mortality, morbidity, length of hospital stay, and additional health care costs.

Compliance with ethical standards This article does not contain any studies with animals performed by any of the authors. Additional informed consent was obtained from all individual participants whose identifying information is included in this article.

Conflict of interest The authors declare that they have no conflicts of interest. 


\section{References}

1. Borgstede JP, Lewis RS, Bhargavan M et al (2004) RADPEER quality assurance program: a multifacility study of interpretive disagreement rates. J Am Coll Radiol 1:59-65

2. Goddard P, Leslie A, Jones A et al (2001) Error in radiology. Br J Radiol 74:949-951

3. Berlin L (2007) Radiologic errors and malpractice: a blurry distinction. AJR Am J Roentgenol 189:517-522

4. Brady A, Laoide RÓ, McCarthy P et al (2012) Discrepancy and error in radiology: concepts, causes and consequences. Ulst Med J 81:3-9

5. Fitzgerald R (2001) Error in radiology. Clin Radiol 56:938-946

6. Caranci F, Brunese L, Reginelli A et al (2012) Neck neoplastic conditions in the emergency setting: role of multidetector computed tomography. Semin Ultrasound CT MR 33(5):443-448

7. Pinto A, Caranci F, Romano L et al (2012) Learning from errors in radiology: a comprehensive review. Semin Ultrasound CT MR 33:379-382

8. Kundel HL, Nodine CF, Carmody D (1978) Visual scanning, pattern recognition and decision-making in pulmonary nodule detection. Invest Radiol 13:175-181

9. Harrigal CL, Erly WK (2007) On-call radiology: community standards and current trends. Semin Ultrasound CT MR 28:85-93

10. Renfrew DL, Franken EA, Berbaum KS et al (1992) Error in radiology: classification and lessons in 182 cases presented at a problem case conference. Radiology 183:145-150

11. Cervo A, Cocozza S, Saccà F et al (2015) The combined use of conventional MRI and MR spectroscopic imaging increases the diagnostic accuracy in amyotrophic lateral sclerosis. Eur J Radiol 84:151-157

12. McCarron MO, Wade C, McCarron P (2014) Optimising neuroimaging effectiveness in a district general hospital. J R Coll Physicians Edinb 44:14-19

13. Cooper VF, Goodhartz LA, Nemcek AA Jr et al (2008) Radiology resident interpretations of on-call imaging studies: the incidence of major discrepancies. Acad Radiol 15:1198-1204

14. Viertel VG, Babiarz LS, Carone M et al (2012) Quality control in neuroradiology: impact of trainees on discrepancy rates. AJNR Am J Neuroradiol 33:1032-1036

15. Babiarz LS, Yousem DM (2012) Quality control in neuroradiology: discrepancies in image interpretation among academic neuroradiologists. AJNR Am J Neuroradiol 33:37-42

16. Yaniv G, Mozes O, Greenberg G et al (2013) Common sites and etiologies of residents' misinterpretation of head CT scans in the emergency department of a level I trauma center. Isr Med Assoc J 15:221-225

17. Walls J, Hunter N, Brasher PM et al (2009) The DePICTORS Study: discrepancies in preliminary interpretation of CT scans between on-call residents and staff. Emerg Radiol 16:303-308

18. Erly WK, Berger WG, Krupinski E et al (2002) Radiology resident evaluation of head CT scan orders in the emergency department. AJNR Am J Neuroradiol 23:103-107

19. Stevens KJ, Griffiths KL, Rosenberg J et al (2008) Discordance rates between preliminary and final radiology reports on crosssectional imaging studies at a level 1 trauma center. Acad Radiol $15: 1217-1226$
20. Filippi CG, Schneider B, Burbank HN et al (2008) Discrepancy rates of radiology resident interpretations of on-call neuroradiology MR imaging studies. Radiology 249:972-979

21. Osborn A (2012) Brain imaging, pathology, anatomy. Lippincott Williams \& Wilkins, Philadelphia

22. Celzo FG, Venstermans C, De Belder F et al. (2013) Brain stones revisited-between a rock and a hard place. Insights Imaging 4(5):625-635

23. Hoe JW (1989) Computed tomography of nasopharyngeal carcinoma. A review of CT appearances in 56 patients. Eur J Radiol 9:83-90

24. Adachi M, Hosoya T, Haku T et al (1998) Dilated VirchowRobin spaces: MRI pathological study. Neuroradiology 40:27-31

25. Bernard JP, Moscoso G, Renier D et al (2001) Cystic malformations of the posterior fossa. Prenat Diagn 21:1064-1069

26. Dimmick SJ, Faulder KC (2009) Normal variants of the cerebral circulation at multidetector CT angiography. Radiographics 29:1027-1043

27. Castillo M (2014) Hystory and evolution of brain tumor imaging: insights through radiology. Radiology 273(2 Suppl):S111-S125

28. Ambrosetto P, Nicolini F, Zoli M et al (2014) Ophthalmoplegic migraine: from questions to answers. Cephalalgia 34(11):914-919

29. Muccio CF, Di Blasi A, Esposito G et al (2013) Perfusion and spectroscopy magnetic resonance imaging in a case of lymphocytic vasculitis mimicking brain tumor. Pol J Radiol 78:66-69

30. Caranci F, Cirillo M, Piccolo D et al (2012) A rare case of intraosseous lipoma involving the sphenoclival region. Neuroradiol $\mathbf{J}$ 25:680-683

31. Smimiotopoulos JG, Murphy FM, Rushing EJ et al (2007) Patterns of contrast enhancement in the brain and meninges. Radiographics 27:525-551

32. Kramer RA (1977) Vermian pseudotumor: a potential pitfall of CT brain scanning with contrast enhancement. Neuroradiology 13(5):229-230

33. Cirillo L, Leonardi M, Dall'Olio M et al (2012) Complications in the treatment of intracranial aneurysms with silk stents: an analysis of 30 consecutive patients. Interv Neuroradiol 18(4):413-425

34. van Wessem KJ, Meijer JM, Leenen LP et al (2011) Blunt traumatic carotid artery dissection: still a pitfall? The rationale for aggressive screening. Eur J Trauma Emerg Surg 37:147-154

35. Pinto A, Brunese L, Pinto F et al (2012) The concept of error and malpractice in radiology. Semin Ultrasound CT MR 33(4):275-279

36. Caranci F, Briganti F, La Porta M et al (2013) Magnetic resonance imaging in brachial plexus injury. Musculoskelet Surg 97:181-190

37. Daffner RH, Wippold FJ II, Bennett DL et al (2009) ACR appropriateness criteria suspected spine trauma. http://www.guideline. gov/content.aspx

38. Grassi R, Rea G, Scaglione M, Brunese L, Scialpi M (2000) Imaging of tracheocele: report of three cases and review of the literature. Radiol Med 100:285-287

39. Lian K, Bharatha A, Aviv RI et al (2011) Interpretation errors in $\mathrm{CT}$ angiography of the head and neck and the benefit of double reading. AJNR Am J Neuroradiol 32:2132-2135

40. Halsted MJ (2004) Radiology peer review as an opportunity to reduce errors and improve patient care. J Am Coll Radiol 1:984-987 